anatomy to compare Comaneci vs balloon assisted catheterization of small feeders.

Methods and Methods Patient is a 37-year-old man who presented with sudden onset of severe headache and left sided weakness. Initial CT head and MRA showed left basal ganglia hemorrhage associated with left thalamic AVM. Using a 3D model of the AVM, 30 iterations of micro-catheterization was performed with balloon (Hyperform 4x7mm), Comaneci Petit (24mm length) stent, and without any device assistance (10 each).

Results During the embolization procedure, Comaneci stent provided adequate support distal to the origin of the arterial feeder to the AVM, and the microcatheter was successfully navigated into the small sharply angled feeder (figure 1A). Experimental model showed similar effectiveness of balloon and Comaneci stent with $3 / 10$ first-attempt success rate for micro-catheterization, and 0/10 without any device (figure 1B). Patient tolerated the procedure well with no ischemic or hemorrhagic complications.

Conclusion Comaneci stent showed similar efficacy compared to a balloon for distal micro catherization without distal flowarrest. Our technical report along with the 3-D model experiments provide insights into the utility of Comaneci vs balloon assisted micro-catherization of the small sharply angled feeders in AVM embolization.

Disclosures H. Saber: None. N. Kaneko: None. S. Tateshima: 2; C; Medtronic, Stryker, Cerenovus. G. Colby: 2; C; Stryker, MicroVention, Medtronic. V. Szeder: None. M. Nour: None. G. Duckwiler: 2; C; Medtronic. R. Jahan: 2; C; Medtronic, Balt.

\section{E-139 COMPREHENSIVE IN-VITRO NEUROVASCULAR MODEL ASSESSMENT FOR IMPLANT SIMULATION}

${ }^{1} \mathrm{~N}$ Norris ${ }^{*},{ }^{2} \mathrm{~K}$ Lewis, ${ }^{2} \mathrm{C}$ Settanni, ${ }^{2} \mathrm{~T}$ Becker, ${ }^{3} \mathrm{~A}$ Ducruet. ${ }^{1}$ Aneuvas Technologies Inc., Flagstaff, $A Z ;{ }^{2}$ Northern Arizona University, Flagstaff, $A Z ;{ }^{3}$ Barrow Neurological Institute, Phoenix, AZ

\subsection{6/neurintsurg-2021-SNIS.234}

Introduction Although current microcatheter technologies have advanced in recent years, corresponding endovascular models still lag behind. Animal models are unable to replicate consistent large and wide neck bifurcation aneurysms with sufficient neurovascular feeders. A system is needed that accurately models vessel tortuosity, flow patterns, detects downstream migration of neurovascular embolic devices, along with displaying and storing peri-procedural and long-term pressure and flow data. This submission utilizes an in-vitro flow model to test the stability of endovascular devices.

Materials and Methods This research brings together clinical, biological, and engineering expertise for the development of benchtop flow models for mechanical assessments of device stability. A full Circle of Willis (CW) in-vitro vessel model was fabricated into vessel analogs using a biomimetic photopolymer, Agilus $30^{\oplus}$ and constructed using a PolyJet ${ }^{\circledast 2}$ (UVcured) 3D-printing additive manufacturing process, capable of replicating accurate anatomical tortuosity. Typical aneurysm positions, verified by a collaborating neuro-interventional surgeon, was 3D-printed at the basilar bifurcation, the posterior communicating (PCA) branch, and at the anterior communicating (ACA) bifurcation (Abstract 139 figure 1).

Results To confirm the usability of the flow model, a novel polymer aneurysm device, NeuroCURE ${ }^{\oplus}$, was deployed under temporary balloon occlusion. The in-vitro model monitored

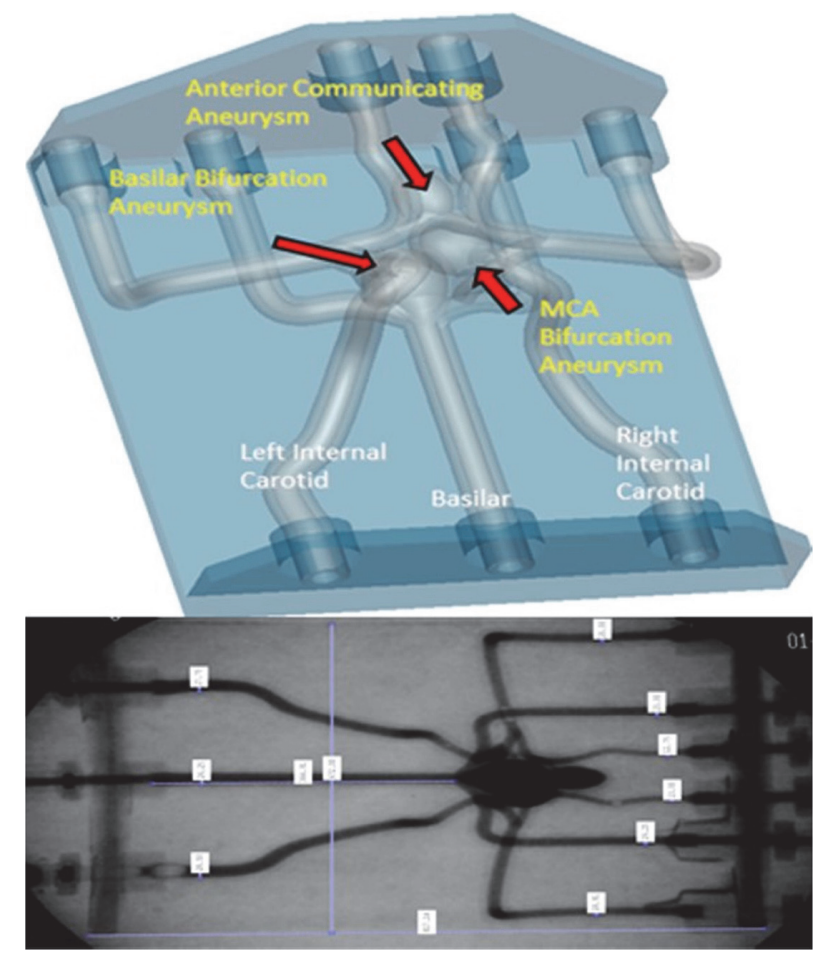

Abstract E-139 Figure 1 CAD model of CW (Top) CW model interior (Bottom) Physiologically-relevant patient flow parameters were tuned within the system using a Super Pump AR (ViVitro Labs). A data acquisition system (DAQ) with LabVIEW ${ }^{\circledR}$ software recorded real time pressure and flow waveforms, mean arterial pressures (MAP) and flows through each inlet and outlet branch of the 3D-printed CW model. The flow model included an introducer for microcatheter and device delivery access

real-time pressure information intra- and post-device delivery. Modified Raymond-Roy (MRR) was used to evaluate posttreatment. The Agilus $30^{\oplus}$ model reproduced a working CW model, and the NeuroCURE device delivery provided validation of the surgical simulation technique. Real-time pressure and flow data provided a greater understanding of flow distribution throughout the CW.

Conclusion This in-vitro aneurysm flow model utilized the latest in UV-cured 3D-printing techniques to provide a realistic simulation of neurovascular tortuosity and aneurysm device delivery. Future additions to the comprehensive flow model include an inline imaging system to quantify any particles (number and size, in accordance with ((USP) XXV <788>) that may be released during endovascular device delivery. Lastly, the model will be a closed-loop sterile system that can run for up to one-month for long-term device integrity testing, complementing in vivo testing of device biocompatibility.

Disclosures N. Norris: None. K. Lewis: None. C. Settanni: None. T. Becker: 1; C; NIH grant\# 5R42NS097069-03. A. Ducruet: None.

\section{E-140 SINGLE CENTER CASE STUDIES COMPRISING OF EARLY CAROTID STENTING IN PATIENTS WHO PRESENT WITH ISCHEMIC STROKES}

${ }^{1} \mathrm{~S}$ Bhatt*, ${ }^{2} \mathrm{M}$ Colasurdo, ${ }^{2} \mathrm{~K}$ Raghuram. 'Department of Neurology, UTMB, Galveston, TX; ${ }^{2}$ Department of Radiology, UTMB, Galveston, TX

10.1136/neurintsurg-2021-SNIS.235 